\title{
Off-critical lattice models and massive SLEs
}

\author{
N. MAKAROV \\ Mathematics 253-37, Caltech \\ Pasadena, CA 91125, USA \\ makarov@caltech.edu \\ S. SMIRNOV \\ Section de mathématiques, Université de Genève \\ 2-4, rue du Lièvre, c.p. 64, 1211 Genève 4, Switzerland \\ E-mail: stanislav.smirnov@math.unige.ch
}

\begin{abstract}
We suggest how versions of Schramm's SLE can be used to describe the scaling limit of some off-critical 2D lattice models. Many open questions remain.
\end{abstract}

Keywords: lattice models, SLE, CFT

\section{Introduction}

During the last 25 years Conformal Field Theory (CFT) was successful in heuristically describing conformally invariant scaling limits of 2D lattice models at criticality, such as the Ising model, percolation, Self-Avoiding Polymers, Potts models, - see Ref. 1 for a collection of the founding papers of the subject.

Recently there was much progress in the mathematical understanding, in large part due to Oded Schramm's introduction of SLEs, or Schramm Loewner Evolutions. $\mathrm{SLE}(\kappa)$ is a one-parameter family of random conformally invariant curves, constructed by running a Loewner Evolution with (real valued) Brownian motion as the driving term. For several models convergence to SLE was established in the scaling limit; moreover, SLE is well-adapted to calculations, which typically boil down to Itô's stochastic calculus. See Ref. 2,3 for an exposition and references.

The key property of SLE is its conformal invariance, which is expected in 2D lattice models only at criticality, and the question naturally arises:

Can SLE success be replicated for off-critical models?

In most off-critical cases to obtain a non-trivial scaling limit one has to adjust some parameter (like temperature in the Ising model or probability of an open site in percolation), sending it at an appropriate speed to the critical value. Such limits lead to massive field theories, so the question can be reformulated as whether one can use SLEs to describe those. Massive CFTs are no longer conformally invariant, but are still covariant when mass is considered as a variable covariant density. 
So far there was limited progress on off-critical SLEs, related work limited to Ref. 4-8. Below we propose an approach based on the combination of SLE Martingale Observables (MO) with potential theory and stochastic analysis, and we start by describing the critical case.

\subsection{SLE and critical lattice models}

Suppose that a critical lattice model defines for every simply connected domain $\Omega$ with two marked boundary points $a, b$ a random discrete simple curve joining them inside $\Omega$. One can think e.g. of a domain wall boundary in the Ising model with Dobrushin boundary conditions or of a LERW - the Loop Erasure of the Random Walk from $a$ to $\partial \Omega \backslash\{b\}$ conditioned on ending at $b$.

The key observation made by Oded Schramm was that if a conformally invariant scaling limit $\gamma$ of the discrete curves exists and satisfies Markov property (i.e. the curve progressively drawn from $a$ to $b$ does not distinguish its past from the boudary of the domain $\Omega$ ), then it can be described by $\operatorname{SLE}(\kappa)$ for some $\kappa \in[0, \infty[$. Take an appropriate time parameterization $\gamma(t)$ and denote by $\Omega_{t}$ the component at $b$ of the domain $\Omega \backslash \gamma[0, t]$. Then the random Loewner conformal map

$$
Z_{t}(z): \Omega_{t} \rightarrow \mathbb{C}_{+}, \quad \gamma(t) \mapsto 0, b \mapsto \infty, \text { normalized at } b
$$

(in what we call a Loewner chart), satisfies the Loewner equation with the Brownian Motion as the driving term. We write it as a stochastic differential equation (SDE)

$$
d Z_{t}(z)=\frac{2}{Z_{t}(z)} d t-d \xi_{t}, \quad d \xi_{t}:=\sqrt{\kappa} d B_{t},
$$

with the SLE driving term $\xi_{t}$ given by the standard Brownian motion $B_{t}$.

Moreover, it turns out that to deduce SLE convergence it is enough to show that just one observable $M_{t}(z)=M\left(z, \Omega_{t}\right)$ (e.g. spin correlation or percolation probability) is conformally covariant and Markov in the limit, see Ref. 3 for a discussion. So far conformal invariance of observables was always estbalished by showing that they are discrete holomorphic (or harmonic) functions of a point satisfying some Boundary Value Problem (BVP) - Dirichlet, Neumann or Riemann-Hilbert.

The covariant holomorphic MOs for SLE can be easily classified, leading to a one parameter family for each $\kappa$ or each spin (conformal dimension) $\sigma$, namely

$$
M_{t}^{\kappa, \sigma, \beta}(z)=M_{t}(z)=Z_{t}(z)^{\beta} Z_{t}^{\prime}(z)^{\sigma}, \quad \text { with } \sigma=\beta+\frac{\beta(\beta-1)}{4} \kappa,
$$

is $(d z)^{\sigma}$-covariant. It is characterized (see Ref. 3) by the Riemann-Hilbert BVP

$$
\bar{\partial} M_{t}=0 \text { in } \Omega_{t}, \quad M_{t}(z) \| \tau^{-\sigma} \text { on } \partial \Omega_{t}, \text { appropriate singularities at } \gamma(t), b .
$$

Note that the MO above can also be rewritten in terms of the complex Poisson kernel in $\Omega_{t}$ at $\gamma(t), P_{t}(z):=-1 / Z_{t}(z)$.

Of special interest is also the family of holomorphic MOs for $\operatorname{SLE}(\kappa)$ :

$$
M_{t}^{\kappa}(z)=M_{t}(z)=\log Z_{t}(z)+\left(1-\frac{\kappa}{4}\right) \log Z_{t}^{\prime}(z) .
$$


Those are covariant pre-pre-Schwarzian forms, and so it suffices to study their imaginary parts - harmonic MOs characterized by

$$
\Delta M_{t}(z)=0 \text { in } \Omega, \quad M_{t}(z)=\arg Z_{t}(z)+\left(1-\frac{\kappa}{4}\right) \arg Z_{t}^{\prime}(z) \text { on } \partial \Omega_{t},
$$

the Dirichlet BVP being well posed and independent of time parameterization, i.e. the choice of normalization at $b$ of the map $Z_{t}(z)$. Such bosonic observables (in Coulomb gas formalism of CFT, they are 1-point functions of the bosonic field in the presence of background charge) feature prominently in Ref. 9-11.

The observables above have the martingale property with respect to corresponding $\operatorname{SLE}(\kappa)$ by a simple application of Itô's calculus: vanishing of their drifts under the diffusion (11) is easy to deduce using (11) and its corollary

$$
d Z_{t}^{\prime}(z)=-\frac{2 Z_{t}^{\prime}(z)}{Z_{t}(z)^{2}} d t
$$

Conversely, if a random curve admits a MO of the mentioned form, similar calculations prove the curve to be a $\operatorname{SLE}(\kappa)$.

\subsection{SLE and off-critical perturbations}

Most discrete holomorphic observables studied in Ref. 10-16 behave well under some off-critical perturbations, leading to discrete Massive Martingale Observable (MMO) satisfying the massive version of the Cauchy-Riemann or Laplace equations:

$$
\begin{aligned}
\bar{\partial}^{(m)} M^{(m)} & :=\bar{\partial} M^{(m)}-i m \overline{M^{(m)}}=0, \\
\Delta^{(m)} M^{(m)} & :=\Delta M^{(m)}-m^{2} M^{(m)}=0,
\end{aligned}
$$

inside $\Omega$ and solving the same boundary value problem as the critical MO. Here mass $m$ can be understood as a function of $z$, changing covariantly under conformal transformations. In discrete setting a power of the lattice mesh enters Eq. (788), thus one has to tend the perturbation parameter to its critical value in a coordinated way with the lattice mesh, so that the mass does not blow up in the scaling limit.

Given a discrete random curve with a discrete MMO, we can ask, to what extent the SLE theory can be applied in the massive case, posing the following problems:

Problem A. Show that discrete MMO has a scaling limit, which is then a MMO for some random curve. Find a SDE for the driving diffusion $d \xi_{t}$ replacing the Brownian motion in the Loewner Evolution (11).

Problem B. Show that this SDE has a unique solution and the corresponding random curve is the scaling limit of the original discrete curves.

Problem C. Use massive SLEs to derive properties of massive field theories and massive SLE curves.

Note that massive models (as well as massive holomorphic functions) usually are even easier to control, so the main problem is the absence of conformal invariance, 
or rather presence of conformal covariance with respect to the mass. Consequently the drift terms in the corresponding diffusions depend on (Euclidean) geometry of the domains constructed dynamically by Loewner evolution, leading to SDEs with general previsible path functionals, which are rather complicated.

Outline of the paper. We were able to advance within the suggested framework, and in Section 2 we present some of our results from the forthcoming Ref. 8. In Section 3 we provide two essential elements of the proof, namely we give an example of the drift computation (in the case of bosonic observables) and establish some simple a priory estimates of the drift (in the $\kappa=4$ bosonic case). Finally we conclude with a list of open questions in Section 4.

Notation. We consider a simply connected domain $\Omega \subset \mathbb{C}$ with two marked points $a, b \in \partial \Omega$, joined by a random Markov curve $\gamma(t)$ with Loewner parameterization. The Loewner map to the half-plane is denoted by $Z_{t}(z)$. We denote by $h$ the harmonic measure, by $G$ the Green's function, and its boundary differentials by

$$
P(u, z)=N_{u} G(\cdot, z), \quad K(u, b)=N_{b} P(u, \cdot)
$$

(the Poisson kernel and Poisson boundary kernel respectively). Here $N_{u}$ stands for the normal derivative at $u$ in the Loewner boundary chart. By $G^{(m)}, P^{(m)}, \ldots$ we denote the corresponding massive objects. The index $t$ signifies that we work in the domain $\Omega_{t}$, i.e. the component at $b$ of $\Omega \backslash \gamma[0, t]$, and $\iint$ denotes the area integral.

\section{Results}

Below we go through all the cases discovered to-date where a lattice model admits a discrete MO with a massive perturbation, and describe results which will appear in Ref. 8. For simplicity we restrict ourselves to the case of constant mass $m$, but the methods should apply to an appropriate class of variable densities $m(z)$.

\subsection{Loop Erased Random Walk with killing, $\kappa=2$}

The LERW converges to $\operatorname{SLE}(2)$, as shown in Ref. 12. The law $\gamma(t)$ of the $\operatorname{LERW}^{(m)}$ on a lattice of mesh $\epsilon$ is defined by applying the same loop erasing procedure to the random walk with a killing rate (i.e. probability to die out at each step)

$$
\delta=m^{2} \epsilon^{2} \text {. }
$$

The RW is done inside a domain $\Omega$ from the boundary point $a$ to the rest of the boundary and conditioned on ending at $b \in \partial \Omega$ - this ensures the Markov property.

Theorem 2.1. For a bounded domain the scaling limit of $L E R W^{(m)}$ exists and is given by the massive SLE(2) with the driving diffusion

$$
\xi_{t}=\sqrt{2} d B_{t}+\lambda_{t} d t, \quad \lambda_{t}=2\left[\log K^{(m)}\left(\cdot, b ; \Omega_{t}\right)\right]^{\prime}\left(\gamma_{t}\right) \quad\left(\text { in the chart } Z_{t}\right) .
$$

The law of the scaling limit is absolutely continuous with respect to SLE(2). 
The proof begins by constructing a discrete harmonic MMO (on appropriate discretization of the domain $\Omega$ ):

$$
M_{t}^{(\delta)}(z) \equiv M^{(\delta)}\left(z ; \gamma(t), b, \Omega_{t}\right):=\frac{G^{(\delta)}(\gamma(t), z ; \partial \Omega \cap \gamma[0, t[)}{h^{(\delta)}(\gamma(t), b ; \partial \Omega \cap \gamma[0, t[)}, \quad z \in \Omega_{t}^{\text {discrete }},
$$

where $G^{(\delta)}$ and $h^{(\delta)}$ denote discrete Green's function and discrete harmonic measure respectively, both with killing rate $\delta$. Then we we fix a boundary chart at $b$ and show that after an appropriate normalizations the discrete MMO converges to

$$
\frac{P_{t}^{(m)}(\gamma(t), z)}{K_{t}^{(m)}(\gamma(t), b)}, \quad\left(z \in \Omega_{t}\right),
$$

a continuous MMO corresponding to (213) with $\beta=-1$ and $\sigma=0$. Now let the driving term $\xi_{t}$ be an Itô process

$$
d \xi_{t}=\lambda(t, \omega) d t+\sigma(t, \omega) d B_{t} .
$$

We claim that if the Loewner chain has MMO (10), then $\sigma \equiv \sqrt{2}$ and $\lambda$ satisfies (9). We now have the equation

$$
d \xi_{t}=\lambda\left(t, \xi_{\bullet}\right) d t+\sqrt{2} d B_{t},
$$

with the previsible path functional $\lambda\left(t, \xi_{\bullet}\right)$ given by (9). We claim that this equation satisfies the standard finiteness condition (cf. Chapter 5 in Ref. 18): $\lambda$ is locally integrable on almost all paths. The question of course arises of existence and uniqueness of SDE solutions. In the case under consideration the answer is quite simple: the path functional $\lambda$ satisfies the Novikov's condition (see Ref. 19)

$$
\mathbb{E}\left[\exp \left(\frac{1}{2} \int_{0}^{\infty} \lambda\left(t, \xi_{\bullet}\right)^{2} d t\right)\right]<\infty
$$

(and in fact, $\int_{0}^{\infty} \lambda\left(t, \xi_{\bullet}\right)^{2} d t \leq$ const $<\infty$ holds for all paths). In particular, the SDE has a unique in law (weak) solution $\xi_{t}$ and its law is absolutely continuous with respect to $\sqrt{2} d B_{t}$. See Proposition 3.2 below for a similar argument. One can then deduce that the massive SLE is the scaling limit of the massive LERW by using the absolute continuity of latter with respect to the LERW.

\subsection{Massive Harmonic Explorer and Gaussian Free Field, $\kappa=4$}

We use the original Harmonic Explorer (HE) construction on a hexagonal lattice, Ref. 10. At each step, the boundary consists of two arcs, and the explorer turns in the direction of one of the arcs with probability equal to its harmonic measure in the current domain $\Omega_{t}$ evaluated at the "growth point." Introducing the killing rate as in the previous section, we get one distinction from the massless case - the two harmonic measures don't sum up to one, so with complementary probability we toss a fair coin to determine the direction of the turn. The MMO is the massive version of the bosonic MO (415) for $\kappa=4$. 
The theory is completely analogous to that of the massive LERW. If the initial domain is bounded, then the scaling limit exists and is absolutely continuous with respect to SLE(4), the scaling limit of massless explorer. We deduce the formula (13) for the drift in more general bosonic case in Proposition 3.1 (for the HE $M_{t}$ is the difference of the massless harmonic measures of the two boundary arcs).

The same framework holds for the massive version of the discrete Gaussian Free Field discussed in Ref. 11, and parts of our construction apply to general bosonic observables.

\subsection{Massive Peano curves, $\kappa=8$}

For a lattice approximation of $\Omega$, we choose its cycle-free subgraph $\Gamma$ with probability proportional to $\alpha^{n(\Gamma)}, n(\Gamma)$ being the number of connected components. Conditioning $\Gamma$ to contain all the edges in the boundary arc $(a, b)$ creates a random Markov interface $\gamma$ from $a$ to $b$ which traces the component wired on the arc $(a, b)$. The case $\alpha=0$ corresponds to the usual Uniform Spanning Tree model, as considered in Ref. 12, whose interface converges to the random Peano curve SLE(8).

The full massive harmonic measure of the boundary with reflection in the unwired part is a discrete MMO, corresponding to (213) with $\beta=1 / 2$ and $\sigma=0$. It has a scaling limit, and we show that the drift has to be

$$
d \lambda_{t}=16\left(\iint_{\Omega_{t}} \tilde{P}_{t} \tilde{P}_{t}^{(m)}\right) d t,
$$

where $\tilde{P}_{t}$ is the minimal (Martin's) kernel for Dirichlet/Neumann boundary conditions in $\left(\Omega_{t}, \gamma(t), b\right)$ and $\tilde{P}_{t}^{(m)}$ its massive counterpart in the Loewner chart.

It would be interesting to interpret the formal expression (12) and show that the SDE is well defined. Note that for $\alpha>0$ the interface is no longer space-filling, thus massive SLE and SLE are mutually singular, while both have scaling dimension 2 .

\subsection{Fortuin-Kasteleyn Ising model, $\kappa=16 / 3$}

The fermionic MO, considered in Ref. 3,13-15,17 for the random cluster representation of the critical Ising model, implies that the interface converges to SLE(16/3). The MO corresponds to (23) with $\beta=-1 / 2$ and $\sigma=1 / 2$, and becomes a MMO under the perturbation by $p$ - the weight of an open edge (FK analogue of magnetization). The techniques of Ref. 15 allow to show the existence of a scaling limit MMO. It solves a Riemann-Hilbert boundary value problem, which makes potential theory and hence derivation of drifts more difficult than in the bosonic case.

\subsection{Critical Ising model, $\kappa=3$}

A similar fermionic MO appears (see Ref. 3,16) in the usual spin representation of the Ising model and implies convergence of the interface (domain wall boundary) to $\operatorname{SLE}(3)$. This is observable (2/3) with $\beta=-1$ and $\sigma=1 / 2$. When perturbed by the 
energy field, it becomes a MMO, which again solves a Riemann-Hilber boundary value problem. While the techniques of Ref. 15 should also be applicable, there are similar difficulties as well.

\section{Techniques}

As an example, we show how to derive the drift and analyze the corresponding SDE for bosonic MOs (4). We work with their imaginary parts, harmonic MOs (5). The corresponding MMOs are massive harmonic (8), while solving the same BVPs.

\subsection{Deriving the drift}

Proposition 3.1. If a random curve is described by a Loewner evolution and has a bosonic MMO satisfying (??), then the driving diffusion is given by

$$
d \xi_{t}=\sqrt{\kappa} d B_{t}+d \lambda_{t}, \quad d \lambda_{t}=\left(\iint_{\Omega_{t}} m^{2} M_{t} P_{t}^{(m)}\right) d t
$$

Remark 3.1. The drift can be rewritten in several ways, e.g. as $\iint_{\Omega_{t}} m^{2} M_{t}^{(m)} P_{t}$.

Proof. Let $M_{t}^{(m)}$ be the massive harmonic MMO solving the BVP (??), then

$$
\Delta^{(m)} M_{t}^{(m)}=0 \Rightarrow \Delta^{(m)}\left(M_{t}^{(m)}-M_{t}\right)=m^{2} M_{t} \Rightarrow M_{t}^{(m)}=M_{t}+m^{2} M_{t} * G_{t}^{(m)},
$$

Similarly for the massive Poisson kernel we have

$$
P_{t}^{(m)}=P_{t}+m^{2} P_{t} * G_{t}^{(m)} .
$$

Describe the massive curve by the Loewner evolution with the driving term $\xi_{t}$

$$
d \xi_{t}=\sqrt{\kappa} d B_{t}+d \lambda_{t} .
$$

Denoting drifts with respect to SLE and massive SLE by $d^{\text {sle }}$ and $d^{\text {msle }}$, we calculate

$$
d^{m s l e} M_{t}=d^{\text {sle }} M_{t}+\operatorname{Im}\left(d \lambda_{t} / Z_{t}\right)=P_{t} d \lambda_{t} .
$$

Using the massive version of the Hadamard's variational formula

$$
d^{m s l e} G_{t}^{(m)}(z, w)=-P_{t}^{(m)}(z) P_{t}^{(m)}(w) d t,
$$

we can write the drift for the MMO:

$$
\begin{aligned}
& 0=d^{m s l e} M_{t}^{(m)}(z) \stackrel{14}{=} d^{m s l e}\left(M_{t}+m^{2} M_{t} * G_{t}^{(m)}\right) \\
& \quad=d^{m s l e} M_{t}+m^{2}\left(d^{m s l e} M_{t}\right) * G_{t}^{(m)}+m^{2} M_{t}(\cdot) *\left(d^{m s l e} G_{t}^{(m)}(z, \cdot)\right) \\
& \stackrel{1617}{=}\left(P_{t}+m^{2} P_{t} * G_{t}^{(m)}\right) d \lambda_{t}-m^{2} M_{t}(\cdot) *\left(P_{t}^{(m)}(z) P_{t}^{(m)}(\cdot)\right) d t \\
& \stackrel{15}{=} P_{t}^{(m)}(z) d \lambda_{t}-P_{t}^{(m)}(z)\left(\iint_{\Omega_{t}} m^{2} M_{t} P_{t}^{(m)}\right) d t,
\end{aligned}
$$

so we deduce (13). 


\subsection{Analysis of diffusion}

Proposition 3.2. In bounded domain $\Omega$ the massive SLE(4) driven by the diffusion (13) for $\kappa=4$ is absolutely continuous with respect to SLE(4).

Proof. First recall that in this case the harmonic MMO is bounded by the maximum principle since $M_{t}^{(m)}=\arg Z_{t} \in[0, \pi]$ on $\partial \Omega_{t}$ by (??). Thus by Remark 3.1

$$
\left|\lambda_{t}\right|=\iint_{\Omega_{t}} m^{2} M_{t}^{m} P_{t} \lesssim \iint_{\Omega_{t}} P_{t} .
$$

Now for a bounded initial domain we can write using the Hadamard's variational formula (17):

$$
\begin{aligned}
\int_{0}^{\infty} \lambda_{t}^{2} d t & \stackrel{\sqrt[18]{\leq}}{\leq} \int_{0}^{\infty} d t \iint_{(z)} P_{t}(z) \iint_{(w)} P_{t}(w) \stackrel{\sqrt[17]{=}}{=}-\int_{0}^{\infty} \iint_{(z)} \iint_{(w)} d G_{t}(z, w) \\
& =\iint_{(z)} \iint_{(w)}\left[G_{0}(z, w)-G_{\infty}(z, w)\right] \leq \iint_{(w)} \iint_{(z)} G_{0}(z, w) \leq C<\infty
\end{aligned}
$$

The last step follows since $\Omega_{0}$ is bounded and so

$$
G_{\Omega_{0}}(z, w) \leq-\log |z-w|+\text { const. }
$$

Applying the Novikov's criterion (11), we conclude that our diffusion is well defined and its law is absolutely continuous with respect to SLE(4).

\section{Questions}

We would like to end with a few questions, which originated in our work.

Question 4.1. How many "physically interesting" (e.g. relevant for the renormalization group) massive perturbations can a CFT have? The arguments above display a one-parameter family of covariant holomorphic MO for each $\operatorname{SLE}(\kappa)$, suggesting that somehow there is a one-parameter family of "canonical" massive perturbations. Are perturbations by non-holomorphic MO also relevant? Are all perturbations generated by MO perturbations?

Question 4.2. Is it always true that diffusion driving a massive version of $\operatorname{SLE}(\kappa)$ is a speed $\kappa$ Brownian motion plus a drift? In the absence of conformal invariance the drift forcibly depends on the geometry of the domain grown. Are all such drifts locally bounded variation path functionals?

Question 4.3. The drifts so far encountered are either absolutely continuous (e.g. LERW and HE cases above) or monotone (e.g. UST case above or percolation perturbations discussed in Ref. 6,7). Are all the possible drifts combinations of those?

Question 4.4. Can one show that a SLE and its massive version are always in the same universality class (in the sense of scaling exponents)? 
Question 4.5. For $\kappa \leq 4$ in our examples the massive and usual SLE are mutually absolutely continuous. Is it true for all perturbations when $\kappa \leq 4$ ?

Question 4.6. Is it it true that for $\kappa>4$ the massive and usual SLE are singular, while being in the same universality class? If not, for which perturbations are they mutually absolutely continuous? We expect the bosonic ones to be among those.

Question 4.7. $\operatorname{SLE}(\kappa)$ almost surely produce simple curves for $\kappa \leq 4$ and curves with double points for $\kappa>4$. "Resampling" the model at a double point alters the curve drastically. Is this the reason for the absolute continuity / singularity dichotomy suggested above?

Question 4.8. Does discrete percolation observable from Ref. 21 have a massive counterpart? If so, which perturbation does it correspond to? This observable requires three marked points, but when two are fused, its continuous counterpart becomes the observable (23) with $\beta=1 / 3$ and $\sigma=0$.

Question 4.9. We have MMOs for at least five different values of $\kappa$. Guess by analogy the driving diffusions for other values of $\kappa$, and show that those are welldefined and lead to random curves.

Question 4.10. In particular, FK Ising MMO from section 2.4 belongs to a family of MMOs arising from (23) with $\sigma=-\beta=-1+8 / \kappa$, cf. Ref. 3. We believe that all those correspond to magnetization perturbations. Is it true, in particular for $\kappa=3$ (spin Ising)? If it holds for $\kappa=6$, can one make a connection with a heuristic formula $d \lambda_{t}=$ const $|d \gamma(t)|^{3 / 4}|d t|^{1 / 2}$ for the drift from Ref. 7?

Question 4.11. Similarly, starting from the spin Ising MMO from section 2.5, we can ask (cf. Ref. 3) whether all MMOs arising from (23) with $\sigma=-\beta / 2=3 / \kappa-1 / 2$ correspond to fugacity perturbations? Note that while LERW MMO from section 2.1 does not belong to this family, its differential does.

Question 4.12. The $O(N)$ model has conjecturally two critical regimes, corresponding to parameter $x$ (bond weight in the loop representation of the high temperature expansion) belonging to $\left\{x_{c}\right\}$ and $] x_{c}, \infty$ [, see Ref. 20. Interfaces conjecturally converge to $\operatorname{SLE}(\kappa)$ and $\operatorname{SLE}(\tilde{\kappa})$ correspondingly, where $\kappa$ and $\tilde{\kappa}$ are known functions of $N$ and satisfy duality (though different from the usual duality $\kappa \kappa^{*}=16$ ):

$$
\frac{1}{\kappa}+\frac{1}{\tilde{\kappa}}=\frac{1}{2}, \quad \kappa \in[8 / 3,4], \tilde{\kappa} \in[4,8] .
$$

As in question 4.11 we expect to have in the first regime a discrete holomorphic MO corresponding to (23) with $\sigma=-\beta / 2=\frac{3}{\kappa}-2$, whose massive counterpart corresponds to perturbation of $x \approx x_{c}$. Then tending mass to $\infty$ we should arrive to the second critical regime. Can we observe this effect in massive SLEs? Namely, does such massive $\operatorname{SLE}(\kappa)$ tend to usual $\operatorname{SLE}(\tilde{\kappa})$ as $m \rightarrow \infty$ ? In particular, does massive SLE(3) tend to SLE(6)? Do other observables lead to different dualities? Can we observe that tending mass to $-\infty$ leads to the frozen regime $x \in\left[0, x_{c}[\right.$ ? 
Question 4.13. Can massive observables (which are in some sense better behaved) help to understand the critical ones? This is the case in Ref. 22, where we establish the conjectured value of the critical temperature for FK model with $q \geq 4$.

\section{Acknowledgments}

This research was supported by the N.S.F. Grant No. 0201893, by the Swiss N.S.F. and by the European Research Council AG CONFRA.

\section{References}

1. C. Itzykson, H. Saleur and J.-B. Zuber (eds.), Conformal invariance and applications to statistical mechanics (World Scientific Publishing Co. Inc., Teaneck, NJ, 1988).

2. O. Schramm, Conformally invariant scaling limits: an overview and a collection of problems, in Proceedings of the International Congress of Mathematicians (Madrid, August 22-30, 2006), (Eur. Math. Soc., Zürich, 2007) pp. 513-543.

3. S. Smirnov, Towards conformal invariance of $2 \mathrm{D}$ lattice models, in Proceedings of the International Congress of Mathematicians (Madrid, August 22-30, 2006), (Eur. Math. Soc., Zürich, 2006) pp. 1421-1451.

4. M. Bauer, D. Bernard and K. Kytölä, J. Stat. Phys. 132, 721 (2008).

5. M. Bauer, D. Bernard, and L. Cantini, J. Stat. Mech. Theory Exp. , P07037, (electronic) (2009).

6. P. Nolin and W. Werner, J. Amer. Math. Soc. 22, 797 (2009).

7. C. Garban, G. Pete and O. Schramm, Work in progress (2008).

8. N. Makarov and S. Smirnov, Massive stochastic Loewner evolutions, In preparation (2009).

9. J. Dubédat, SLE and the free field: Partition functions and couplings, Preprint (2007).

10. O. Schramm and S. Sheffield, Ann. Probab. 33, 2127 (2005).

11. O. Schramm and S. Sheffield, Acta Math. 202, 21 (2009).

12. G. F. Lawler, O. Schramm and W. Werner, Ann. Probab. 32, 939 (2004).

13. S. Smirnov, Conformal invariance in random cluster models, I, Ann. of Math. (2) (to appear).

14. V. Riva and J. Cardy, J. Stat. Mech. Theory Exp., P12001, (electronic) (2006).

15. D. Chelkak and S. Smirnov, Universality in the 2D Ising model and conformal invariance of fermionic observables, Preprint (2009).

16. D. Chelkak and S. Smirnov, Conformal invariance of the 2D Ising model at criticality, Preprint (2009).

17. S. Smirnov, Conformal invariance in random cluster models, II, Preprint (2009).

18. L. C. G. Rogers and D. Williams, Diffusions, Markov processes, and martingales. Vol. 2 (Cambridge University Press, Cambridge, 2000).

19. B. Øksendal, Stochastic differential equations, (Springer-Verlag, Berlin, 2003).

20. W. Kager and B. Nienhuis, J. Statist. Phys. 115, 1149 (2004).

21. S. Smirnov, C. R. Math. Acad. Sci. Paris 333, 239 (2001).

22. V. Beffara, H. Duminil-Copin and S. Smirnov, The self-dual point of the 2D randomcluster model is critical above $q=4$, Preprint (2009). 